\title{
ENSAIOS
}

Denis Renato Oliveira denis.oliveira@ufla.br Professor Adjunto do Departamento de Administração Pública da Universidade Federal de Lavras (UFLA). Lavras, $M G, B R$.

João Luiz Passador jlpassador@usp.br Professor Titular do Departamento de Administração da Faculdade de Economia Administração e Contabilidade de Ribeirão Preto da Universidade de São Paulo. São Paulo, SP, BR.

Silvia Ines Dallavalle de Padua dallavalle@usp.br Professora Associada do Departamento de Administração da Faculdade de Economia Administração e Contabilidade de Ribeirão Preto da Universidade de São Paulo. São Paulo, SP, BR.

Diego César Terra de Andrade diego.terra@ifsuldeminas.edu.br Doutor em Administração. Professor Associado do Instituto Federal de Educação, Ciência e Tecnologia do Sul de Minas. Pouso Alegre, $M G, B R$.

\section{GESTÃO DO CONHECIMENTO, CULTURA ORGANIZACIONAL E GESTÃO DE PESSOAS COM A GESTÃO DE PROCESSOS E QUESTÕES ORGANIZACIONAIS EMERGENTES: UMA ANÁLISE CRÍTICA DA DINÂMICA SUBJETIVA EM GESTÃO POR PROCESSOS (BP)}

\author{
KNOWLEDGE MANAGEMENT, \\ ORGANIZATIONAL CULTURE AND \\ PEOPLE MANAGEMENT WITH PROCESS \\ MANAGEMENT AND EMERGING \\ ORGANIZATIONAL ISSUES: A CRITICAL \\ ANALYSIS OF SUBJECTIVE DYNAMICS IN \\ PROCESS MANAGEMENT (BP)
}

\section{RESUMO}

A maioria dos trabalhos desenvolvidos em gestão por processos tem-se limitado a explorar os problemas da relação entre a organização funcional e a perspectiva de processo com ênfase nos aspectos técnicos de sua aplicação. A literatura de base conceitual que versa sobre os aspectos humanos contemplados em sua operacionalização ainda é insipiente (TARHAN; TURETKEN; REIJERS, 2016). Tendo em vista a importância da dinâmica subjetiva no processo de análise organizacional, objetiva-se promover uma discussão teórica sobre a relação entre gestão por processos, cultura organizacional e gestão do conhecimento, concentrando esforços no entendimento das relações humanas que podem influenciar o desempenho organizacional. Conclusivamente, há fortes indícios de que, apesar dos altos investimentos e sofisticação dos sistemas, a abordagem do empurrar, comumente adotada, não gera valor aos negócios da organização. O problema fundamental reside no modelo subjacente, mecânico, de processamento de informação, que se adota na gestão do conhecimento. Este nega a natureza socialmente construída do conhecimento. Nesse sentido, a gestão de processos intensiva em conhecimento confirma uma necessidade emergente de adoção da estratégia de "puxar" como modelo de gestão, uma vez que ela incorpora processos organizacionais baseados na combinação sinérgica de dados e a capacidade de processamento das informações pela tecnologia, as quais são suportadas pela capacidade criativa e inovadora dos seres humanos. 
Palavras-chave: Gestão por Processos. Cultura Organizacional. Gestão do Conhecimento.

\section{ABSTRACT}

Most of the work developed in process management has been limited to exploring the problems of the relationship between the functional organization and the process perspective with emphasis on the technical aspects of its application. The conceptual-based literature that deals with the human aspects contemplated in its operation is still incipient (TARHAN; TURETKEN; REIJERS, 2016). Given the importance of subjective dynamics in the process of organizational analysis, the objective is to promote a theoretical discussion on the relationship between process management, organizational culture and knowledge management, focusing efforts on the understanding of human relationships that may influence organizational performance. Conclusively, there are strong evidences that, despite the high investments and sophistication of the systems, the push approach commonly adopted does not add value to the organization's business. The fundamental problem lies in the underlying model, mechanical, of information processing that is adopted in knowledge management. It denies the socially constructed nature of knowledge. In this sense, knowledge-intensive process management confirms an emerging need to adopt the "pull" strategy as a management model. One that incorporates organizational processes based on the synergistic combination of data and the capacity of information to be processed by technology. These are supported by the creative and innovative capacity of human beings.

Keywords: Processes Management. Organizational Culture. Knowledge Management.

\section{INTRODUÇÃO}

Os softwares utilizados na operacionalização e no gerenciamento de processos são considerados, segundo Contador et al.
(2005), como um dos principais facilitadores da gestão do conhecimento referente ao processo, oportunizando maior facilidade para a obtenção, a distribuição e a análise dos dados. Esses softwares que colaboram com a gestão por processos são denominados de Business Process Management (BPM). Entre as funcionalidades que podem ser disponibilizadas por meio desses softwares, está a de aumentar a capacidade dos gestores e dos demais envolvidos com o processo em estabelecer uma troca de imagens e ideias. Segundo Nonaka e Takeuchi (2005), é nesse ínterim que ocorre a geração do conhecimento.

Embora um grande destaque tenha sido dado à questão da gestão por processos nas organizações contemporâneas (ANTONUCCI; GOEKE, 2011; GLYKAS, 2011; GONZÁLEZ et al., 2010; KOHLBACHER, 2010; MUNSTERMANN; ECKHARDT; WEITZEL, 2010; SMART; MADDERN; MAULL, 2009), sobretudo quando se relaciona os ganhos econômicos decorrentes de sua aplicação, a grande maioria dos trabalhos desenvolvidos tem-se limitado a explorar e a descrever as implicações da implantação dessa disciplina nas organizações. Esse destaque existe quando se consideram os problemas da relação entre a organização funcional e a perspectiva de processo (PALMBERG, 2010).

Posto isso, a crítica que se faz recai sobre a ênfase dada aos aspectos técnicos da aplicação da gestão por processos, com foco restrito na definição dos níveis de análise, categorização dos processos e técnicas de mapeamento, documentação e mensuração de desempenho. A própria literatura na área está fundamentada nas tentativas de se explicar o "como fazer" (HELLSTRÖM; PETERSON, 2005; KÜNG; HAGEN, 2007; SANDHU; GUNASEKARAN, 2004; ONGARO, 2004). Entretanto, ainda, há poucos estudos de base conceitual que versam sobre as questões organizacionais de execução; especificamente, quando se trata dos aspectos humanos contemplados em sua operacionalização.

Embora deva fornecer ferramentas, téc- 
nicas e relações que se enquadram em ambas as categorias (funcionais, informacionais e dinâmicas ou comportamentais), as metodologias existentes não conseguem integrar os conceitos de gestão de processos, gestão de recursos humanos e fluxo de trabalho. Faltam, também, métricas de negócio e análise de índices qualitativos, o que demonstra a necessidade de abordagens holísticas de medição de desempenho e articulação (SKERLAVAJ et al., 2007; ELBASHIR; COLLIER; DAVERN， 2008; HERZOG; TONCHIA; POLAJNAR, 2009). De acordo com Busch e Fettke (2011), existem poucas pesquisas que tratam da intersecção entre gestão por processos e gestão do conhecimento. Para Marjanovic (2010), o problema da integração entre os temas é desafiador.

Nesse sentido, busca-se responder à seguinte questão de pesquisa: como são contemplados os elementos culturais e a gestão do conhecimento nas discussões sobre gestão por processos? Para isso, objetiva-se promover uma discussão teórica sobre a relação entre gestão por processos, cultura organizacional e gestão do conhecimento. A tentativa é contribuir para o maior entendimento da dinâmica subjetiva da gestão por processos, embora os esforços sejam concentrados na análise dos critérios intangíveis que influenciam o desempenho organizacional.

O estudo se baseará em: (1) uma pesquisa sistemática em bases de dados internacionais (Scopus, Science Direct e Web-of-Science) por artigos que tragam à tona a proposta de ciclo de vida BPM e a gestão do conhecimento, fazendo menção às palavras-chave, "business process management" e "knowledge management"; (2) uma análise das referências dos artigos selecionados no passo anterior que ainda não tenham sido contemplados; (3) priorização de publicações realizadas a partir do ano de 2008; (4) uso das ferramentas de ordenação por relevância disponibilizadas pelos sites adotados e; (5) aplicação de filtro de relevância subjetivo por meio de análise das obras, buscando publicações que relacionam KM, BPM e Cultura.

$\mathrm{O}$ presente artigo estrutura-se em quatro seções. Inicialmente, além desta introdução, discutem-se brevemente as dimensões operacionais da orientação por processos. Na seção dois, apresenta-se uma discussão sobre os pressupostos básicos da gestão do conhecimento e da cultura organizacional enquanto critérios subjetivos de análise. Na seção três, faz-se uma análise crítica sobre a dinâmica de integração dos aspectos relacionais nos estudos sobre gestão por processos. E, por último, faz-se um resumo das principais contribuições desta pesquisa para o "estado da arte" em BPM.

\section{DIMENSÕES OPERACIO- NAIS DA ORIENTAÇÃO POR PROCESSOS (OP)}

A concepção de "processos" em negócios se refere às atividades de gestão que perpassam os limites funcionais de estruturas hierárquicas e se apresentam como uma nova dinâmica de geração de valor ao cliente (REIJERS, 2006; TARHAN; TURETKEN; REIJERS, 2016). Nesse sentido, destaca-se o desenvolvimento de técnicas que proveem suporte às funções específicas por meio da integração dos sistemas para o controle do trabalho, a obtenção de informações e a mensuração do desempenho (PRADABWONG et al., 2017).

Uma organização orientada para o processo (OP) deve aplicar, de forma abrangente, o conceito de gestão por processos de negócios (BPM). A melhor descrição para isso está fundamentada na realização da missão organizacional por meio da sistematização dos processos, traduzindo-os em atividades e tarefas (ABPMP, 2009). O BPM não se preocupa apenas com o projeto, sua implantação e execução, também se dedica à interação, ao controle, análise e à otimização dos processos de negócio (SMITH; FINGAR, 2003; HARMON, 2019).

A operacionalização de BPM envolve a observação de diversas dimensões. Entre elas, destacam-se:

1. detalhamento dos processos de negócios e suas inter-relações (HINTERHUBER, 1995; KASEMSAP, 2017); 
2. apoio da alta gerência (HAMMER, 2007a);

3. existência de gerentes (HAMMER; STANTON, 1999);

4. ferramentas de medição de desempenho (HAMMER, 2007b);

5. adaptação cultural, tratando-se do trabalho em equipe (WILLAERT et al., 2007), disponibilidade para mudança (HAMMER, 2007a), orientação para o cliente (REIJERS, 2006), estilo de liderança cooperativa (HAMMER, 2007a), etc.;

6. adoção de tecnologia da informação (KIRCHMER, 2009);

7. adaptação da estrutura organizacional (REIJERS, 2006); 2007a);

8. gestão do conhecimento (HAMMER,

9. adoção de sistemas de RH (HAMMER, 2007a; WILLAERT et al., 2007); e,

10. instância formal de coordenação e integração de todos os projetos.

Todo processo de sistematização da prática do BPM, entretanto, deve ser feita com base em uma análise detalhada de processo. A compreensão do ambiente de negócios, a cultura e o contexto organizacional específico. Essas são as métricas de desempenho e as consequências de sua mudança com base no conhecimento, no nível de envolvimento humano e comprometimento funcional. Todas são questões essenciais para que se consiga mostrar a eficiência do negócio em alcançar seus objetivos e criar um entendimento de como o trabalho ocorre e/ou deveria ocorrer na organização (ABPMP, 2009).

Caracterizada como um ciclo de vida contínuo de atividades integradas, a prática de BPM contempla as etapas de planejamento, análise, desenho e/ou modelagem, implantação, monitoramento, controle e refinamento. À medida que os processos de negócio se movem nessa direção, perpassando por fases evolutivas, são habilitados ou restringidos por uma variedade de fatores intangíveis que incluem os sistemas de valores, as crenças, a cultura e o conhecimento, todos envoltos pela questão do comprometimento organizacional (ABPMP, 2009).

O ciclo de vida do BPM vai ao encontro do modelo clássico do ciclo de vida de desenvolvimento de softwares (figura 1), conforme proposto por Pressman (2006).

Figura 1 - Ciclo de vida clássico do desenvolvimento de software

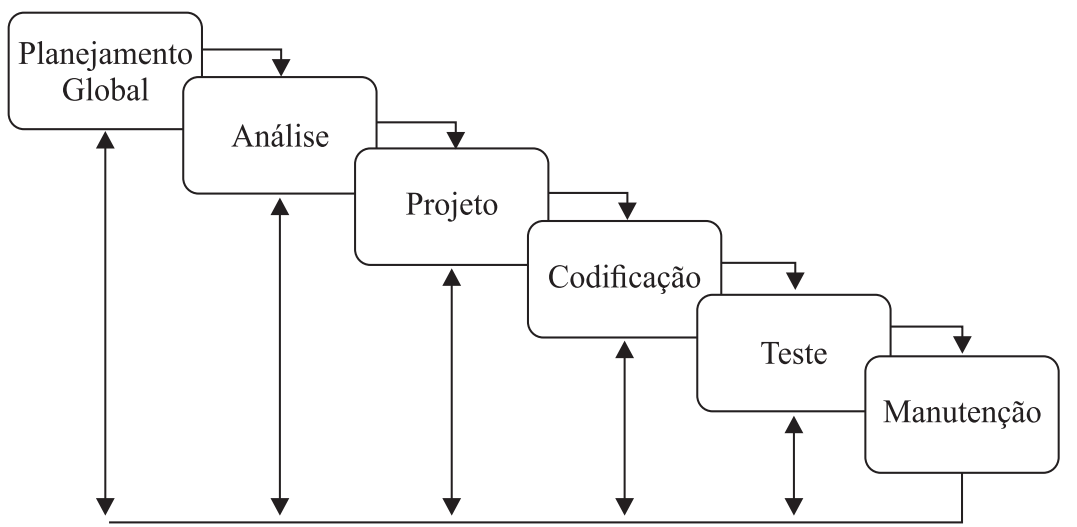

Fonte: adaptado de Pressman (2006, p. 33).

A fase de planejamento tem como finalidade definir o escopo do software, bem como suas restrições e conceitos. Na fase de análise, o objetivo é elaborar as documentações lógicas que serão utilizadas nos processos de confecção, nos protótipos e relatórios necessários. $\mathrm{Na}$ fase de plane- jamento, estabelece os elementos de hardware e software necessários para a execução do projeto.

Desse modo, o processo de desenvolvimento do software exige a confecção de documentos para auxiliar no levantamento e na definição das funcionalidades do cliente ao qual 
devem ser implantadas ao novo sistema (PRESSMAN, 2006; GONÇALVES et al., 2016).

\section{GESTÃO DO CONHECI- MENTO E CULTURA ORGA- NIZACIONAL: CRITÉRIOS SUBJETIVOS DE ANÁLISE}

A gestão do conhecimento se refere ao desenvolvimento de sistemas e processos que visam à criação, à identificação, à integração, à recuperação, ao compartilhamento e à utilização do conhecimento dentro da empresa. Ela volta-se para a criação e a organização de fluxos de informação entre os vários níveis organizacionais. Seu objetivo é gerar, incrementar, desenvolver e partilhar o conhecimento dentro da organização. Possibilitando, assim, o aumento do aprendizado individual e grupal. Dessa forma, pode criar competências exclusivas que diferenciarão a organização no mercado em que compete (RÉ et al., 2007; BAJAJ; SANGWAN, 2017).
De acordo com Nonaka e Takeuchi (2008), a construção do conhecimento ocorre no processo de conversão de conhecimento tácito em conhecimento explícito. Esses são componentes dicotômicos e, aparentemente, opostos. O conhecimento explícito trata das especificações formais ou recursos que podem ser facilmente processados, transmitidos ou sistematicamente armazenados pelos indivíduos. Devido à sua natureza subjetiva, o conhecimento tácito não pode ser processado ou transmitido por qualquer método lógico, pois trata-se de um processo intuitivo, que envolve o sistema de valores e a experiência individual das pessoas. Desse modo, para que possa ser compartilhado e crie valor para a organização, o conhecimento tácito precisa ser convertido em conhecimento explícito (CHOO, 2003; RODRIGUES et al., 2017).

Ainda conforme Nonaka e Takeuchi (2008), esse processo de conversão ocorre de quatro maneiras (figura 2):

Figura 2 - Processo de conversão do conhecimento

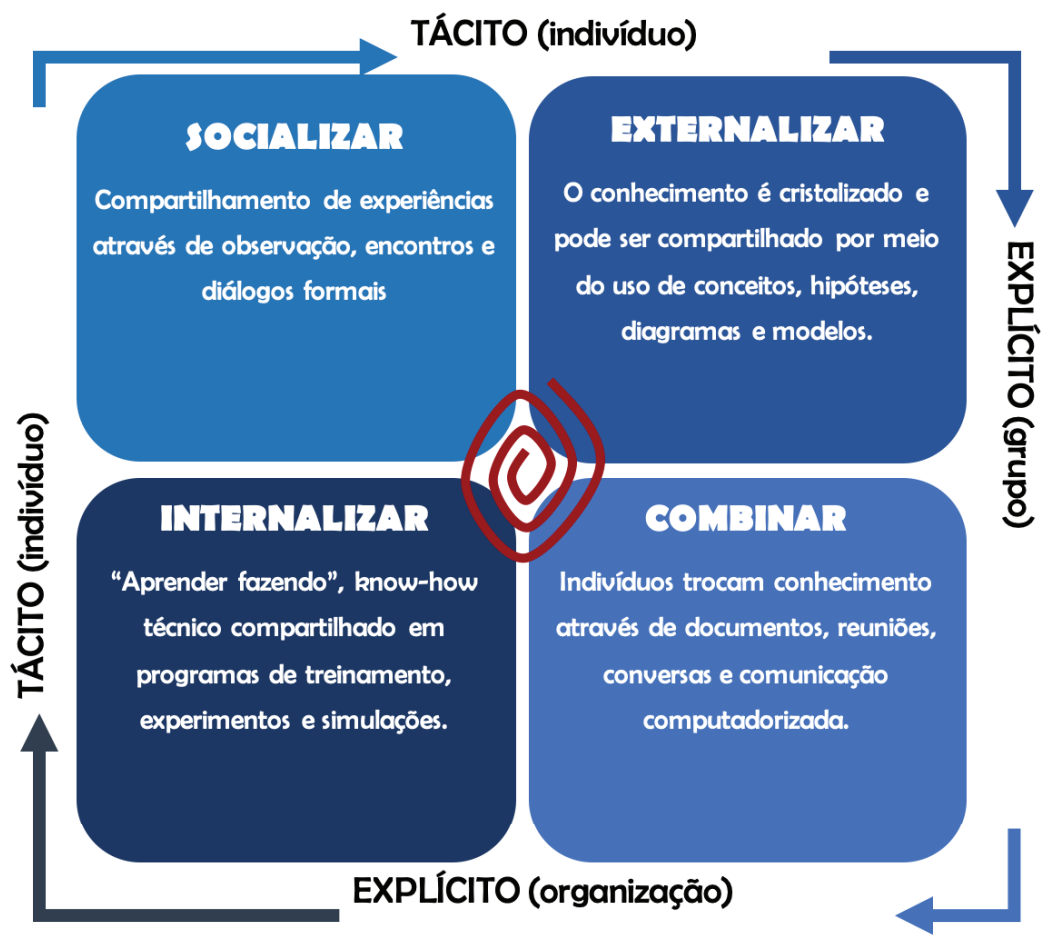

Fonte: adaptado de Nonaka e Takeuchi (2008). 
Pedagogicamente, tem-se:

1. Socialização, que consiste no compartilhamento e criação do conhecimento tácito por meio da experiência direta (conversão de conhecimento tácito em conhecimento tácito).

2. Exteriorização, que trata da articulação do conhecimento tácito por meio do diálogo e da reflexão (conversão de conhecimento tácito em conhecimento explícito).

3. Combinação, que contempla a sistematização e aplicação do conhecimento explícito e informação (conversão de conhecimento explícito em conhecimento explícito).

4. Internalização, quando ocorre o aprendizado por meio da prática intensiva (conversão de conhecimento explícito em conhecimento tácito).

Perucci e Campos (2017) e Choo (2003) afirmam que esse processo de conversão se retroalimenta em uma espiral contínua de construção do conhecimento organizacional.

É nesse contexto que se impõe e se observa o que fora conceito de aprendizagem organizacional. Esse é caracterizado pelo desenvolvimento da capacidade de mudança e adaptação contínua do ambiente, em que as pessoas expandem, continuamente, sua capacidade de criar os resultados que desejam. É onde surgem novos e elevados padrões de raciocínio, em que a aspiração coletiva é libertada, e as pessoas aprendem, continuamente, a aprender em grupo (SENGE, 1990; ROCHA-PINTO, 2015). A transformação de uma organização aprendiz envolve o estabelecimento de uma estratégia que vise ao replanejamento da estrutura e o remodelamento da cultura organizacional (ROBBINS, 2008; THOMAS; PETERSON, 2016).

\section{ANÁLISE CRÍTICA DA DINÂ- MICA DE INTEGRAÇÃO DOS ASPECTOS HUMANOS EM BPM}

A preocupação comum após o surgimento do BPM se refere à visão de automatização dos processos transacionais por meio da padronização de atividades. No entanto, os limites de BPM têm-se expandido para acomodar a di- mensão do conhecimento, da experiência e da criatividade, critérios intangíveis dificilmente imitados pelos concorrentes (EL SAWY, JOSEFEK, 2003; GÁBOR et al., 2016).

Esta abordagem holística está vinculada à procura de estratégias para melhor aproveitamento do capital humano; e é nesse sentido que têm sido evocadas questões subjetivas, como a gestão de conhecimento e cultura organizacional, relativamente não estruturadas, ou seja, de difícil mensuração.

Contudo, o desafio está no fato de o conhecimento organizacional derivar de muitas formas diferentes. Ele não advém apenas por meio de documentos ou modelos formais, mas também das rotinas, dos processos e das práticas organizacionais que independem da padronização de atividades em BPM. O conhecimento é uma combinação de experiência, contexto, interpretações e reflexões e envolve mais a participação humana que a própria informação (MARJANOVIC, 2010; LITTLE; DEOKAR, 2016), uma vez que essa última pode ser limitada ou facilitada pelo perfil cultural presente nas organizações.

De acordo com Machlup (1962) e Druker (1999), o conhecimento utilizado pelas organizações é produzido por profissionais que são apontados como trabalhadores de conhecimento, os quais, por meio de suas competências individuais, produzem sistemas de gestão, de origem tecnológica ou estratégica.

Trabalhadores de conhecimento dentro das empresas possuem um papel de grande importância para viabilizar a criação e a transferência de conhecimento. De acordo com Boff e Abel (2005) e Machado, Cabral e Vaccaro (2018), são esses profissionais que transformam informação em insumo, combinando-a com seu conhecimento individual com o objetivo de produzir nova informação como resultado de sua atividade.

É a partir dessas características que é possível determinar, dentro das empresas, quem são os profissionais de conhecimento, podendo-se, ainda, levar em consideração os diversos níveis de especialização. Assim, tor- 
na-se necessário um melhor entendimento das características desse tipo de profissional. Destarte, é essencial identificar quais competências o diferenciam de um trabalhador tradicional e quais recursos por eles são utilizados para potencializar sua escala produtiva.

O grande desafio, portanto, está relacionado com o processo de captura de conhecimento, bem como o conhecimento adquirido em si. Por muitos anos, as pesquisas em BPM sobre a prática de gestão do conhecimento seguem a estratégia de "empurrar" (MALHOTRA, 2005; MAO et al., 2016). Esse modelo era baseado na ideia de que a tecnologia poderia ser usada para capturar, codificar e armazenar o conhecimento e, em seguida, transferi-lo para o usuário no momento certo. No entanto, há fortes indícios de que, apesar dos altos investimentos e sofisticação dos sistemas, essa abordagem não gera valor aos negócios da organização (MALHOTRA, 2005). O problema fundamental reside no modelo subjacente, mecânico, de processamento de informação que se adota na gestão do conhecimento (MALHOTRA, 2005), uma vez que ele nega a natureza socialmente construída do conhecimento (NEWELL et al., 2002; GHERARDI; MIELE, 2018).

Além disso, uma das principais razões para que os esforços de gestão do conhecimento sejam separados das atividades cotidianas é a imprecisão dos sistemas de coleta de informações utilizados para a transformação de conhecimento tácito em conhecimento explícito (PFEFFER; SUTTON, 2000). Enquanto o co- nhecimento explícito (processo) pode ser capturado e armazenado, o conhecimento tácito ou experimental, que inclui o know-how, os modelos mentais e as práticas organizacionais complexas, é impossível de ser capturado em sua totalidade. Por isso, o processo de codificação do conhecimento tácito mais eficiente em uma organização é geralmente limitado à interação das pessoas que possuem um conhecimento local (DAVENPORT; PRUSAK, 1998; COLOMBELLI et al., 2019).

O trabalho de conhecimento é definido pela variedade ao invés da rotina de execução das atividades, pela exigência de profissionais de alto nível de habilidade e experiência (BOFF; ABEL, 2005). Segundo a definição desses autores, é possível se concluir que, em uma mesma área de atuação, existem dois tipos de profissionais: profissionais de conhecimento e profissionais tradicionais; estes executam suas atividades de forma rotineira e previsível; enquanto profissionais de conhecimento são capazes de interpretar e processar as informações de suas tarefas em determinadas situações, inclusive, as críticas, o que os possibilita produzir novas informações para resolução de problemas e aumento de experiência. Os profissionais de conhecimento possuem um papel de destaque dentro das empresas, atuando em diversos cargos.

Tais papéis são classificados por Boff e Abel (2005) por meio dos profissionais que geram conhecimento, conforme quadro 1 .

Quadro 1 - Classificação dos profissionais que criam conhecimento

\begin{tabular}{|c|c|c|}
\hline Criadores de conhecimento & Atividades & Exemplos de trabalhadores \\
\hline Operadores & $\begin{array}{l}\text { Acumulam e geram conhecimento tácito; } \\
\text { Habilidade adquiridas com a experiência }\end{array}$ & Profissionais da linha de frente \\
\hline Especialistas & $\begin{array}{l}\text { Acumulam, geram e atualizam } \\
\text { conhecimento explícito }\end{array}$ & $\begin{array}{l}\text { Cientistas, planejadores, } \\
\text { analistas }\end{array}$ \\
\hline Engenheiros & $\begin{array}{l}\text { Transformam conhecimento tácito em } \\
\text { explícito e vice-e-versa }\end{array}$ & Gerentes de nível médio \\
\hline Gerentes & $\begin{array}{l}\text { Gerenciam todo o processo de criação de } \\
\text { conhecimento na empresa }\end{array}$ & Gerentes de nível sênior \\
\hline
\end{tabular}

Fonte: adaptado de Boff e Abel (2005, p. 3). 
Nesse sentido, a gestão de processos intensiva em conhecimento confirma uma necessidade emergente de adoção da estratégia de "puxar" como modelo de gestão. Esse novo modelo de gestão incorpora os processos organizacionais baseados na combinação sinérgica de dados e a capacidade de processamento das informações a tecnologia suportadas pela capacidade criativa e inovadora dos seres humanos (MALHOTRA, 2005; MAO et al., 2016).

Essa estratégia, no entanto, deve partir das pessoas e de sua capacidade criativa e proativa de geração de valor, que é restringida ou facilitada pelas características culturais presentes na organização. Ou seja, não há como dissociar as políticas de gestão do conhecimento dos estudos sobre o perfil cultural da organização e dos recursos humanos que compõem seu quadro funcional.

Baseado no uso da epistemologia de Karl Popper, Dalamaris et al. (2007) afirmam que ainda não está claro como integrar a gestão do conhecimento na gestão de processos de negócio, sobretudo a julgar pelo limitado número de trabalhos publicados nesse tema.

Nesse sentido, por exemplo, Little e Deokar (2016) e Marjanovic (2007) afirmam que, em tempos recentes, a necessidade premente de competir com base no conhecimento, em vez de automação de processos, ampliou a área de Gestão de Processos de Negócios (BPM) para incluir os processos de negócios intensivos em conhecimento. Para corroborar suas afirmações, o autor enfoca a integração da BPM e Gestão do Conhecimento (KM) no contexto de processos de negócios voltados para o cliente. O caso confirma a importância do conhecimento relacionado com o processo, em especial, a sua relação com a estratégia competitiva da organização.

Smith e Mckeen (2004), no entanto, apontam que "gestores do conhecimento" estão repensando como e onde o conhecimento realmente agrega valor, pois, conforme apontam Marjanovic e Seethamaraju (2008), os ingredientes principais da diferenciação competitiva das organizações são o conhecimento humano, a experiência e a criatividade. Como já apontado, esses não podem ser padronizados, prescritos e facilmente adquiridos. Nesse sentido, esse tipo de estudo não possibilita replicações possíveis.

Ademais, Sanikar e Deokar (2010) afirmam que, com a finalidade de obter vantagem competitiva sustentável na economia do conhecimento de hoje, as organizações estão olhando para além de processos de workflow transacional de rotina para apoiar os processos de conhecimento intensivo. Os sistemas tradicionais de gestão de processos de negócios são eficazes na prestação de apoio à coordenação. No entanto, não são voltadas para a prestação de apoio e conhecimentos relevantes. Além disso, os sistemas de gestão do conhecimento são utilizados de uma forma ad hoc, sem, explicitamente, serem ligados aos processos organizacionais subjacentes.

Sistemas de gestão do conhecimento com base em processo (PKM) surgiram como uma solução potencial para apoiar os processos de conhecimento intensivo; mas, as diretrizes de design para o desenvolvimento de sistemas de PKM são mínimas. E, para tal, buscam discutir a viabilidade desse tipo de projeto, concluindo que isso é possível, ou seja, é factível de estudo e replicação de um ambiente organizacional, em que a cultura esteja voltada a essa intersecção.

Amavardi e Lee (2005) corroboram tal afirmação e acrescentam que os ativos de conhecimento de uma empresa incluem sua estrutura, cultura, processos, funcionários e artefatos físicos. Para esses autores, apesar de a literatura de gestão do conhecimento (KM) ter a tendência de enfatizar o conhecimento dos colaboradores como um locus de esforços de gestão do conhecimento, esse ponto de vista é, perfeitamente, racional e justificável. Os autores ainda acrescentam que, também, há uma quantidade considerável de conhecimentos incorporados em procedimentos operacionais da empresa.

Nesse sentido, Amavardi e Lee (2005) e Marjanovic (2010) defendem a visualização do conhecimento organizacional a partir dessa perspectiva e afirmam ser possível gerir uma estrutura capaz de alavancar a gestão do processo de conhecimento, partindo de uma definição, a 
classificação dos processos e uma caracterização do processo de geração de conhecimento. Amavardi e Lee (2005) fornecem sete dimensões por meio das quais o conhecimento do processo pode ser visto, são elas: estrutura de pessoal e de coordenação, de desempenho e ferramentas, discursivas, resultados, qualidade e implicações. Todos destinados a servir como um ponto de partida para gerir o conhecimento do processo.

\section{REFLEXÕES E CONTRIBUIÇÕES}

O objetivo da gestão por processos é assegurar a melhoria contínua do desempenho da organização por meio da elevação dos níveis de qualidade. As práticas de gestão do conhecimento aplicadas na operacionalização de BPM são vistas como elemento importante na identificação de problemas e oportunidades. No entanto, precisam estar relacionadas com uma análise crítica da cultura organizacional existente.

Pensar em gerenciamento de processos de negócio é pensar, constantemente, em mudanças sobre "quando", "o quê" e "como" implantar melhorias. Essa retórica contextualista pode remeter à análise dos aspectos metodológicos e fazer pensar se, realmente, existe uma receita ou sequenciamento específico para a definição e o desenho de processos. O que se pode observar é que os autores tentam transformar o conhecimento tácito em explícito como se fosse necessário padronizar as atividades e/ou tarefas. No entanto, destaca-se que isso infere maior atenção e precisa ser mais bem investigado, principalmente quando se considera um modelo de gestão baseado em contingências.

Embora seja evidente a necessidade de adoção de práticas formais no gerenciamento de processos como forma de compartilhar conhecimento, questiona-se, com base na leitura crítica dos estudos sobre BPM, de que forma isso pode ser feito sem levar em consideração a questão do impacto social que provoca as mudanças, sobretudo quando se observa o desempenho organizacional. Essas mudanças apenas ganham notoriedade quando discutidas e anali- sadas sob o enfoque econômico, em termos de ganhos de produtividade.

Quando se faz uma análise específica sobre a importância da gestão por processos, em detrimento da estrutura funcional, visualiza-se pouca preocupação com os aspectos culturais e como esses elementos serão considerados. Em qualquer processo de mudança, especificamente quando se consideram os riscos e os problemas envolvidos, deve-se levar em consideração a questão da capacidade de adaptação organizacional e aceitação social das alterações introduzidas.

Quando Fleury e Fischer (1989) analisam a cultura organizacional, referem-se ao conjunto de valores, expressos em elementos simbólicos e em práticas organizacionais, que, em sua capacidade de ordenar, atribuir significações, construir a identidade organizacional, tanto agem como elementos de comunicação e consenso, como expressam e instrumentalizam as relações de dominação.

Para Schein (1992), a cultura é o que diferencia as organizações na forma como novos membros aprendem a maneira correta de perceber, pensar e sentir-se em relação aos problemas que afetam a organização. No entanto, é preciso observar que a aprendizagem não pode ser forçada, apressada ou imposta aos indivíduos. Isso porque há a tendência humana de apropriação do conhecimento (SENGE, 1999). Essa regra, frequentemente, passa despercebida aos gestores na ânsia de transformar a organização em uma organização que aprende.

A cultura de uma organização é um sistema de valores e crenças compartilhados que influenciam (e não condicionam) o comportamento daqueles que os compartilham (RÉ et al., 2007). Cada organização desenvolve sua própria cultura, produzindo sua identidade, que se manifesta por meio dos padrões de comportamento assumido pelos funcionários, regendo sua conduta.

Para alterar a cultura, necessita-se implantar uma estratégia de gestão do conhecimento de "empurrar", motivando as pessoas a buscar a aprendizagem continuada (BITENCOURT, 2004). Isso pressupõe que os indivíduos dentro 
da organização tenham liberdade para questionar e alterar suas práticas, convicções e valores, ou seja, que a aprendizagem e a inovação contínuas sejam valores básicos da organização.

Nesse sentido, cabe ao gestor de BPM selecionar e atribuir papéis àqueles que irão liderar e gerenciar a equipe (analistas ou especialistas) para assegurar que o projeto seja desenvolvido e que a análise seja necessária e suficiente na representação do estado do processo. Esse esforço individual é o passo inicial para que o comprometimento organizacional seja criado, e uma possível garantia de sucesso na condução das atividades seja alcançada (ABPMP, 2009).

No entanto, antes de se iniciar um projeto de análise, devem ser determinados o escopo do projeto e as estruturas e ferramentas a serem utilizadas, entre as diversas possibilidades existentes. Cabe ao gestor escolher o processo por meio da identificação dos objetivos-chave do negócio e das métricas de desempenho a serem utilizadas; definir o escopo de profundidade da análise e escolher as estruturas analíticas de trabalho.

Para Lawler III, Boudreau e Mohrman (2006), por mais útil que seja a necessidade de avaliação ou medição de desempenho em organizações, os estudos sempre esbarram na questão intangível do processo de análise, mais especificamente, em sua aplicabilidade quando se consideram os impactos sobre os resultados econômico-financeiros. Para ser um parceiro estratégico, os executivos precisam ter conhecimento das estratégias de negócios, desenho e mudança organizacional; ainda, precisam saber como integrar as práticas de gestão a essas estratégias para que possam suportar os desenhos organizacionais. Efetivamente, deparando-se com essas mudanças, as áreas funcionais devem focar quanto elas podem agregar de valor na empresa e quais os resultados que isso pode gerar.

Para a maioria das empresas, a compatibilidade entre os indicadores monitorados não reflete bem os vetores estratégicos essenciais para a criação de valor. Isso se trata do principal problema: incongruência entre o que é medido e o que é importante. Portanto, pode-se consi- derar, hoje, que o novo paradigma econômico, caracterizado pela velocidade, pela inovação, pela curta duração do tempo dos ciclos, pela qualidade e pela satisfação dos clientes, acentua a importância dos ativos intangíveis, que são o conhecimento, a inovação e, sobretudo, a cultura organizacional. Essa nova concepção talvez seja o marco para essa nova estrutura de modelagem de processos.

Há de se destacar tanto a subjetividade do campo organizacional quanto a questão objetiva dos indicadores de desempenho quando o assunto é a integração de critérios subjetivos na operacionalização das práticas de BPM. Encontrar as causas e os feitos de um problema no âmbito organizacional não é tão simples como seguir uma sequência de passos. Portanto, deve-se considerar a competência dos indivíduos que fazem a pesquisa e seu entendimento conceitual e lógico da realidade que se pretende estudar.

\section{REFERÊNCIAS}

ABPMP. Association of Business Process Management Professionals. Guia para Gerenciamento de Processos de Negócio: Corpo Comum de Conhecimento. (BPM CBOK). [S.l.], 2009.

AMAVARDI, C. S.; LEE, L. The dimensions $f$ process knowledge. Knowledge and Process Management, v. 12, n. 1, p. 65-76, 2005.

ANTONUCCI, Y. L.; GOEKE, R. J. Identification of appropriate responsibilities and positions for business process management success. Business Process Management Journal, v. 17, n. 1, p. 127-146, 2011.

BAJAJ, Anu; SANGWAN, Om Prakash. Aspect oriented software testing. In: INTERNATIONAL CONFERENCE ON CLOUD COMPUTING, DATA SCIENCE \& ENGINEERING-CONFLUENCE, 7., 2017, India. Proceedings [...]. India: IEEE, 2017. p. 809-814.

BITENCOURT, C. (org.). Gestão contempo- 
rânea de pessoas: novas práticas, conceitos tradicionais. Porto Alegre: Bookman, 2004.

BOFF, L. H.; ABEL, M. Autodesenvolvimento e Competências: o caso do trabalhador de conhecimento como especialista. In: RUAS, R. et al. Aprendizagem Organizacional e Competências. Porto Alegre: Bookman, 2005.

BUSCH, P.; FETTKE, P. Business process management under the microscope: the potential of social network analysis. In: Hawaii International Conference on System Sciences, 44., 2014, Koloa, Kauai, HI. Proceedings [...]. Koloa, Kauai, HI: HICSS, 2011.

CHOO, C. W. A organização do conhecimento: como as organizações usam a informação para criar significados, construir conhecimento e tomar decisões. São Paulo: SENAC, 2003.

COLOMBELLI, Alessandra et al. To what extent do young innovative companies take advantage of policy support to enact innovation appropriation mechanisms?. Research Policy, p. $103797,2019$.

CONTADOR, J. C. et al. Gestão do conhecimento aplicada à gestão por processos: identificação de funcionalidades requeridas às soluções de business process management system (BPMS). Revista de Administração e Inovação, São Paulo, v. 2, n. 2, p. 5-18, 2005.

DALAMARIS, P. et al. A framework for the improvement of knowledge-intensive business process. Business Process Management Journal, v. 13, n. 2, 2007.

DAVENPORT, T.; PRUSAK, L. Working knowledge: how organisations manage what they now. Boston, Massachusetts: Harvard Business School Press, 1998.

DRUCKER, Peter Ferdinand. Desafios gerenciais para o século XXI. São Paulo: Pioneira, 1999.
EL SAWY, O. A.; JOSEFEK, R. A. Business process as nexus of knowledge. In: HOLSAPPLE, C. (ed.). Handbook on Knowledge Management. [S.l]: Knowledge Matters, Springer, 2003.

ELBASHIR, M. Z.; COLLIER, P. A.; DAVERN, M. J. Measuring the effects of business intelligence systems: the relationship between business process and organizational performance. International Journal of Accounting Information Systems, v. 9, n. 3, p. 135-153, 2008.

FLEURY, M. T. L.; FISCHER, R. M. (coord.). Cultura e poder nas organizações. São Paulo: Atlas, 1989.

GÁBOR, András et al. Corporate knowledge discovery and organizational learning: the role, importance, and application of semantic business process management - the ProKEX case. In: GÁBOR, András; KÕ, Andrea (ed.). Corporate Knowledge Discovery and Organizational Learning. Cham: Springer, 2016. p. 1-31.

GHERARDI, Silvia; MIELE, Francesco. Knowledge Management from a Social Perspective: The Contribution of Practice-Based Studies. In: SYED, J. et al. (ed.). The Palgrave Handbook of Knowledge Management. Cham: Palgrave Macmillan, 2018. p. 151-176.

GLYKAS, M. M. Effort Based Performance Measurement in Business Process Management. Knowledge and Process Management, v.18, n. 1, p.10-33, 2011.

GONÇALVES, Rodrigo Franco et al. Uma abordagem sistêmica do processo de produção em engenharia web, na fase de concepção. Production, v. 26, n. 2, p. 402-416, 2016.

GONZÁLEZ, L. S. et al. Measurement in business processes: a systematic review. Business Process Management Journal. Bradford, v. 16, n. 1, p. 114-134, 2010. 
HAMMER, M. The process audit. Harvard Business Review, v. 85, n. 4, p.111-123, 2007a.

HAMMER, M. The 7 deadly sins of performance measurement. MIT Sloan Management Review, v. 48, p. 19-28, 2007 b.

HAMMER, M.; STANTON, S. How process enterprises really work. Harvard Business Review, v. 77, n. 6, p. 108-118, 1999.

HARMON, Paul. Business process change: a business process management guide for managers and process professionals. [S.l.]: Morgan Kaufmann, 2019.

HELLSTRÖM, A.; PETERSON, J. Becoming process-oriented: a study on organizational transformation. In: QMOD CONFERENCE, 8., 2005, Palermo. Proceedings [...]. Palermo, 2005 .

HERZOG, N. V.; TONCHIA, S.; POLAJNAR, A. Linkages between manufacturing strategy, benchmarking, performance measurement and business process reengineering. Computers \& Industrial Engineering, v. 57, n. 3, p. 963975, 2009.

HINTERHUBER, H. H. Business process management: the European approach. Business Change \& Re-engineering, v.2, n. 4, p. 63-73, 1995.

KASEMSAP, Kijpokin. Unifying a framework of organizational culture, organizational climate, knowledge management, and job performance. In: ORGANIZATIONAL Culture and Behavior: concepts, methodologies, tools, and applications. [S.l.]: IGI Global, 2017. p. 327355.

KIRCHMER, M. High Performance through Process Excellence. Berlin: Springer, 2009.

KOHLBACHER, M. The effects of process orientation: a literature review. Business Pro- cess Management Journal. Bradford, v.16, n. 1, p. 135-152, 2010.

KÜNG, P.; HAGEN, C. The fruits of business process management: an experience report from a Swiss bank. Business Process Management Journal, v. 13, n. 4, p. 477-487, 2007.

LAWLER III, E. E.; BOUDREAU, J. W.; MOHRMAN, S. A. with AliceYee Mark, Beth Neilson and Nora Osganian. Achieving Strategic Excellence: an assessment of Human Resource Organizations. Stanford, California: Stanford Business School, 2006.

LITTLE, Todd A.; DEOKAR, Amit V. Understanding knowledge creation in the context of knowledge-intensive business processes. Journal of Knowledge Management, v. 20, n. 5, p. 858-879, 2016.

MACHADO, Clarice Lucas da Silva; CABRAL, Patricia Martins Fagundes; VACCARO, Guilherme Luis Roehe. Technology Companies Analyzed Based on the Multidimensional Work Motivation Scale/Segmento de Tecnologia Analisado pela Multidimensional Work Motivation Scale. RAC-Revista de Administracao Contemporanea, v. 22, n. 3, p. 336-355, 2018.

MACHLUP, Fritz. The production and distribution of knowledge in the United States. [S.l.]: Princeton University Press, 1962.

MALHOTRA, Y. Integrating knowledge management technologies in organizational business process: getting real time enterprises to deliver real business performance. Journal of Knowledge Management, v. 9, n. 1, p. 7-28, 2005.

MAO, Hongyi et al. Information technology resource, knowledge management capability, and competitive advantage: The moderating role of resource commitment. International Journal of Information Management, v. 36, n. 6, p. 1062-1074, 2016. 
MARJANOVIC, Olivera. The next stage of operational business intelligence: Creating new challenges for business process management. In: ANNUAL HAWAII INTERNATIONAL CONFERENCE ON SYSTEM SCIENCES (HICSS'07), 40., 2007, Waikoloa, HI, USA. Proceedings [...]. Waikoloa, HI, USA: IEEE, 2007. p. 215c-215c.

MARJANOVIC, O. A case study of BPM and $\mathrm{KM}$ integration: from process automation to knowledge intensive business process. In: INT. CONF. ON INFORMATION TECHNOLOGY INTERFACES, 32., 2010, Cavtat, Croatia. Proceedings [...]. Cavtat, Croatia, 2010.

MARJANOVIC, O.; SEETHAMRAJU, R. Understanding knowledge-intensive, practice-oriented business process. In: ANNUAL HAWAII INTERNATIONAL CONFERENCE ON SYSTEM SCIENCES, 41., 2008, Waikoloa, Big Island. Proceedings [...]. Waikoloa, Big Island, HI: IEEE Computer Society, 2008.

MARJANOVIC, Olivera. A case study of BPM and $\mathrm{KM}$ integration: from process automation to knowledge intensive business processes. In: INTERNATIONAL CONFERENCE ON INFORMATION TECHNOLOGY INTERFACES, 32., 2010, Croatia. Proceedings [...]. Croatia: IEEE, 2010. p. 237-242.

MUNSTERMANN, B.; ECKHARDT, A.; WEITZEL, T. The performance impact of business process standardization: An empirical evaluation of the recruitment process. Business Process Management Journal, v. 16, n. 1, p. 29-56, 2010.

NEWELL, S. et al. Managing Knowledge Work. [S.l.]: Palgrave, 2002.

NONAKA, I.; TAKEUCHI, H. Gestão do Conhecimento. Trad. Ana Thorell. Porto Alegre: Bookman, 2008.

ONGARO, E. Process management in the pub- lic sector: the experience of one-stop shops in Italy. The International Journal of Public Sector Management, v. 17, n. 1, p. 81-107, 2004.

PALMBERG, Klara. Experiences of implementing process management: a multiple-case study. Business Process Management Journal, v. 16, n. 1, p. 93-113, 2010.

PERUCCI, Camilo Cesar; CAMPOS, Fernando Celso de. The relationship of the learning school and the cultural school strategies with processes of agile software production/A relacao da escola estrategica de aprendizado e da escola cultural com processos de producao de software agil. Revista Exacta, v. 15, n. 3, p. 395-407, 2017.

PFEFFER, J.; SUTTON, R. The knowing-doing gap. Boston, MA: Harvard Business School Press, 2000.

PRADABWONG, Jiraporn et al. Business process management and supply chain collaboration: effects on performance and competitiveness. Supply Chain Management: An International Journal, v. 22, n. 2, p. 107-121, 2017.

PRESSMAN, R. S. Engenharia de software. São Paulo: Makron Books, 2006.

RÉ, C. A. T. et al. The impact of organizational culture in knowledge management process. In: XXVII ENCONTRO NACIONAL DE ENGENHARIA DE PRODUÇÃO, 27., 2007, Foz do Iguaçu. Anais [...]. Foz do Iguaçu: ENEGEP, 2007.

REIJERS, H. A. Implementing BPM systems: the role of process orientation. Business Process Management Journal, v. 12, n. 4, p. 389409, 2006.

ROBBINS, S. P. Organizational Behavior. 10. ed. São Paulo: Prentice Hall, 2008. 
ROCHA-PINTO, Sandra Regina da. Dimensões funcionais da gestão de pessoas. [S.l.]: FGV, 2015.

RODRIGUES, Adriana Alves et al. Desafios da gestão de dados na era do big data: perspectivas profissionais. Informação e Tecnologia (ITEC), Marília/João Pessoa, v. 4, n. 2, p. 6379, 2017.

SANDHU, M.; GUNASEKARAN, A. Business process development in project-based industry. Business Process Management, v. 10, n. 6, p. 673-690, 2004.

SANIKAR, S.; DEOKAR, A. Knowledge Management Systems for Knowledge Intensive-Process: Design Approach and Illustrative Example. In: ANNUAL HAWAII INTERNATIONAL CONFERENCE ON SYSTEM SCIENCES, 43., 2010, Waikoloa, Big Island. Proceedings $[\ldots]$. Waikoloa, Big Island, HI: IEEE Computer Society, 2010.

SCHEIN, E. H. Organizational culture and leadership. San Francisco: Jossey-Bass, 1992.

SENGE, P. A quinta disciplina. São Paulo: Círculo do Livro, 1990.

SENGE, P. A dança das mudanças. Rio de Janeiro: Campus, 1999.

SKERLAVAJ, M. et al. Organizational learning culture: the missing link between business process change and organizational performance. International Journal of Production Economics, v. 106, n. 2, p. 346-367, 2007.

SMART, P. A.; MADDERN, H.; MAULL, R. $\mathrm{S}$. Understanding Business Process Management: Implications for Theory and Practice. British Journal of Management, v. 20, p. 491-507, 2009.

SMITH, H.; FINGAR, P. Business Process Management: The Third Wave. Meghan-
Kiffer Press, Tampa, FL, 2003.

SMITH, H.; McKEEN, J. D. Developments in practice XII: Knowledge-enabling business process. Comm. Of AIS, v. 13, p. 25-38, 2004.

TARHAN, Ayca; TURETKEN, Oktay; REIJERS, Hajo A. Business process maturity models: A systematic literature review. Information and Software Technology, v. 75, p. 122-134, 2016.

THOMAS, David C.; PETERSON, Mark F. Cross-cultural management: essential concepts. Sage Publications, 2016.

WILLAERT, P. et al. The process-oriented organization: a holistic view. In: DADAM, Alonso. P.; ROSEMANN, M. (ed.). BPM 2007, LNCS 4714. Berlin: Springer, 2007. p. 1-15. 